\title{
Charge Density Determination for Al-rich Composition L1o-ordered gamma-TiAl by Convergent Beam Electron Diffraction
}

\author{
Xiahan Sang ${ }^{1,2}$, Andreas K. Kulovits ${ }^{1,3}$, Guofeng Wang ${ }^{1}$ and Jörg M.K. Wiezorek ${ }^{1}$ \\ 1. Department of Mechanical Engineering and Materials Science, University of Pittsburgh, \\ Pittsburgh, PA, USA, 15261 \\ 2. Department of Materials Science and Engineering, North Carolina State University, \\ Raleigh, NC, USA, 27606 \\ 3. Department of Materials Science and Engineering, Carnegie Mellon University, Pittsburgh, PA, USA, \\ 15213
}

The electron density difference map, $\Delta \rho(\mathrm{r})=\rho(\mathrm{r})_{\text {Cryst }}-\rho(\mathrm{r})_{\text {IAM }}$, here defined as the difference between the electron density of a crystal, $\rho(\mathrm{r})_{\text {Cryst }}$, and that of the equivalent independent atom model (IAM), $\rho(\mathrm{r})_{\text {IAM, }}$ represents one of the quantum mechanical characteristics central for developing fundamental understanding of materials. Convergent beam electron diffraction (CBED) experiments performed with modern transmission electron microscope (TEM) instruments equipped with energy filter and charge coupled devices (CCD) permit probing of nano-scale volumes of perfect crystal and have been shown to enable the measurements of low-order structure factor, $F_{g}$, with sufficient accuracy to obtain $\Delta \rho(r)$ for 3d-electron system transition metals (e.g. $\mathrm{Cr}, \mathrm{Fe}, \mathrm{Co}, \mathrm{Ni}$ ) and some binary intermetallic phases (e.g. $\mathrm{NiAl}, \mathrm{TiAl}$ and FePd) [1, 2]. The facility to determine experimentally the $\Delta \rho(\mathrm{r})$ for these d-electron materials warrants use of the CBED measurements as additional metrics in validation of density functional theory (DFT) calculations [2].

Using multi-beam off-zone axis (MBOZA) CBED diffraction geometries sets of multiple Fg and the Debye Waller factors have been determined here for the chemically ordered intermetallic phase $\gamma$-TiAl with slightly Al-rich off-stoichiometric composition, i.e., Ti-52at\%Al. The lattice parameters of the offstoichiometric $\gamma$-TiAl phase, with the tetragonal $\mathrm{L}_{0}$ structure, and the elemental composition have been determined by combining X-ray diffraction, locally resolved CBED HOLZ line analyses [3] and X-ray energy dispersive spectroscopy using binary standards [2, 4]. Experimental details and the dynamical diffraction theory based iterative refinements for the CBED data quantifications have been described previously $[1,2]$. It has been shown for equiatomic $\gamma$-TiAl that DFT calculations based on the local density approximation (LDA) fail to treat accurately the 3d-electron system related bonding effects, while using the generalized gradient approximations (GGA) achieved considerably improved match and qualitatively excellent agreement with experimentally determined low-order $\mathrm{F}_{\mathrm{g}}$ and $\Delta \rho(\mathrm{r})[2,4]$. However, GGA DFT calculations predicted larger electron charge delocalization than is observed in the CBED based experimentally determined $\Delta \rho(\mathrm{r})$ for equiatomic $\gamma$-TiAl [4]. Figure 1 shows the difference between the X-ray structure factors, $F_{g}$, determined here for the two different composition TiAl phase crystals and the IAM based X-ray structure factors. Based on sets of the first five low-order structure factors, $F_{g}$, those with Miller indices $h k l$ satisfying $h^{2}+k^{2}+l^{2}<5$, charge density maps, $\Delta \rho(r)$, have been obtained from the CBED experiments and GGA DFT calculations (e.g. Fig. 2). Comparison of the $\Delta \rho(\mathrm{r})$ obtained from CBED and DFT indicated that effects from the small (2at.\%) Al-excess in the binary intermetallic phase $\gamma$-TiAl are most discernible for the compositionally all-Ti planes, e.g. the (001)sections (Fig. 2). Incorporating the excess $\mathrm{Al}$ by substitution on $\mathrm{Ti}$ sites appears to enhance delocalization of charge density between second nearest neighbor Ti atoms along $<010\rangle$, while reducing it for nearest neighbor Ti atom bonds along $<110>$ (Fig. 2). 


\section{References}

[1] XH Sang, AK Kulovits and JMK Wiezorek, Acta Crystallographica A66 (2010) p. 694

[2] XH Sang, AK Kulovits, GF Wang and JMK Wiezorek, J. Chemical Physics 138 (2013) p.084504

[3] Tanaka, M., Terauchi, M, Convergent Beam Electron Diffraction, JEOL, Tokyo (1985) p1.

[4] XH Sang, AK Kulovits, GF Wang and JMK Wiezorek, Philosophical Magazine 92 (2012) p.4408

[5] The authors acknowledge support from the Office of Basic Energy Sciences, Division of Materials Science and Engineering (Grant No DE-FG02-08ER46545).

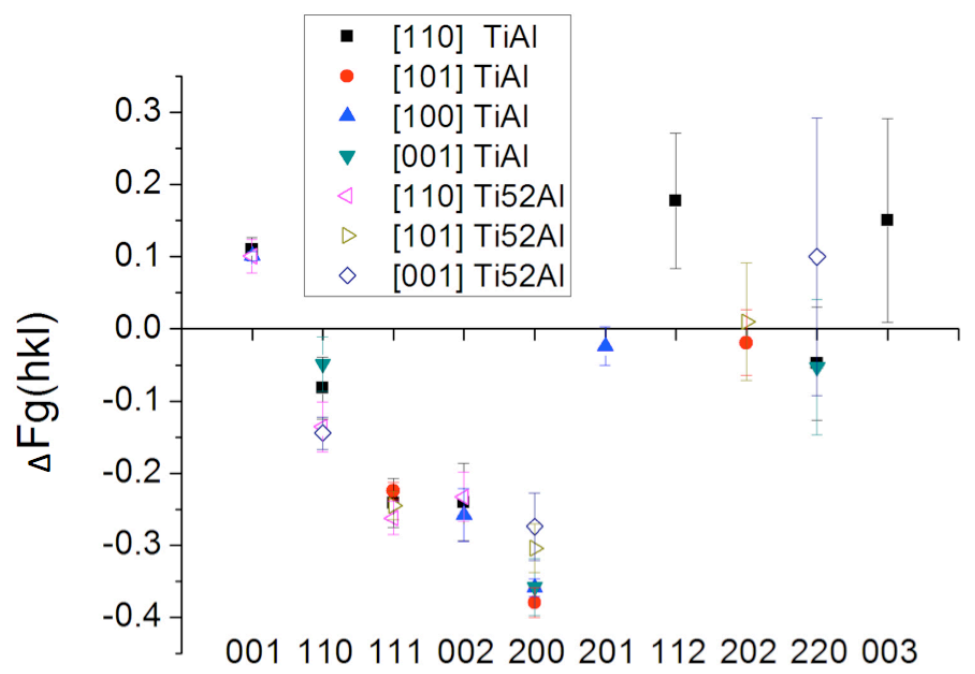

FIG. 1: Difference betweenthe experimentally determined X-ray structure factors and the IAM structure factors, $\Delta \mathrm{Fg}$, for equiatomic (TiAl) and slightly Al-rich offstoichiometric (Ti-52Al) $\gamma$ - TiAl phase. The hkl are plotted in increasing order, from 001 to 003 , along the abscissa.

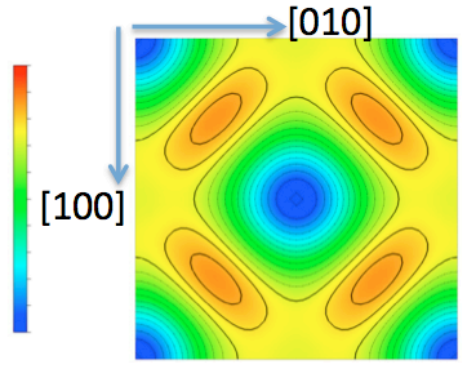

CBED: Ti-50Al

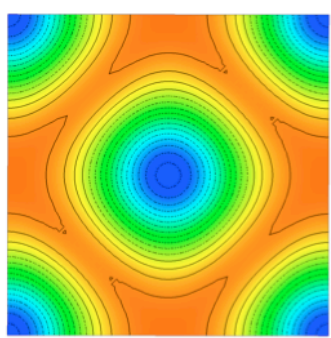

CBED: Ti-52Al

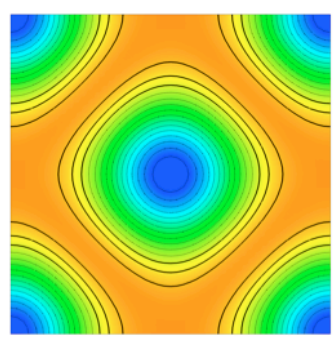

GGA DFT: Ti-50AI

FIG. 2: Example $\Delta \rho(\mathrm{r})$ sections in (001), all Ti plane, of L10-structure tP4 unit cell for CBED experiment derived and DFT calculated equiatomic Ti50Al and Al-rich Ti-52Al. 